\title{
Signal discriminability, S-R compatibility and choice reaction time'
}

With a very simple display, signal discriminability and $S-R$ compatibility interact in their effects on choice RT. This result is discussed in the context of previous demonstrations of interactions between a number of different variables affecting choice RT.

A type of display very commonly used in choiceresponse tasks is a matched set of signal lamps arranged in a line horizontal across S's field of view. It is usually assumed that whichever of these lamps may be lit a human $S$ will take the same time to recognize the fact. This assumption is important if we wish to use data from choice reaction time (RT) experiments to select between currently fashionable functional models for the ways in which Ss may code signals into responses: e.g., whether by successive dichotomizations between members of set of signals (Hick, 1952), by search through a cognitive set of signals in memory (Fitts \& Switzer, 1962; Sternberg, 1964), or by parallel or seriatum tests on signal attributes (Egeth, 1966). Consequently, before using a particular display to test the effect of other variables on choice RT it was expedient to discover how far it could be assumed that all the signals on the display were equally easy to discriminate.

In all the models for choice-responding which we have mentioned, any systematic effect may be taken into consideration if we know the nature of its interactions with the other variables which we wish to study. In all these models signal discriminability may interact with other variables in two possible ways:

(1) Independent input and output uncertainty: Variations in the Ss' uncertainty about the perceptual identification of a signal need not interact with uncertainty concerning the production of a motor response to it. Various functional schemes may be hypothesized in order to meet this requirement. For example, $s$ may make a verbal (or other) mediating response defining the state of the display, and then select an overt response appropriate to this mediating label. Here any increase in the time required to identify the signal (and hence to make the mediating response) will not affect the time taken to select an appropriate motor act (Stermberg, 1964). Discrimination-time will thus be a variable added to the time required by other processes contributing to observed RT. That is, in descriptive equations of the type $R T=a+b$ ( $\log$ No. of stimulus and response alternatives) discrimination-time will be subsumed under the additive function, $a$, rather than under the multiplicative function, $b$.
(2) Related input and output uncertainty: Alternatively, difficulties encountered at any stage in the processes involved in the identification of a signal and the production of a response may interact in some more complex manner than simple addition to bring about variations in choice $R T$.

An experiment was made to determine whether systematic variations in RT were associated with the relative positions of eight signal lamps on a displayand if so, how this effect interacted with S-R compatibility.

Method

A stimulus presenting and response-timing apparatus (SPARTA) $^{1}$ was programmed to present a sequence of 300 successive signals by lighting one of eight neon signal lamps at a time in random order. These lamps were set in horizontal line centered on a $3 \mathrm{ft}$. $x 3 \mathrm{ft}$. display panel. Each lamp exposed a flat circular surface $1 / 4 \mathrm{in}$. in diameter, flush with the panel. Lamps were 2.75 in. apart edge to edge.

Twenty six men and 10 women visitors to an exhibition $^{1}$ where SPARTA was on display served as Ss. They were from 17 to 36 years old. Each $S$ sat with his head 24 in. from the center of the display, resting his hands on a keyboard. Eighteen Ss answered any of the left hand lamps (1 through 4) by pressing a single key with their left hand, and answered any of the right hand lamps (5 through 8 ) by pressing another key with their right hand (Good Compatibility group). The remaining $18 \mathrm{Ss}$ reversed the allocation of keys to lamps (Poor Compatibility group).

The display always changed within $20 \mathrm{msec}$, of any response. All Ss therefore ran through a sequence of 300 successive signals and responses in a selfpaced task as fast and accurately as possible. SPARTA punched out codes identifying each signal in turn, the response made to it (whether correct or wrong), and the elapsed time since the last response to within .01 sec.

\section{Results}

Output was printed and scanned by eye to locate errors. The Good Compatibility group (GC) made $3.4 \%$ errors and the Poor Compatibility group (PC) made $4.9 \%$ of errors $(t=2.91, p<.01)$. Every error, and the three responses following it, were eliminated from the data. Means of each S's mean RT to each signal bulb were calculated from the remaining correct responses and are plotted in Fig. 1:

An analysis of variance showed significant differences in RT between groups $(p<.01)$ and between lamps $(p<.01)$. The interaction-term for Groups vs. 
$\triangle$ G.C.CONDITION

- P.C.CONDITION

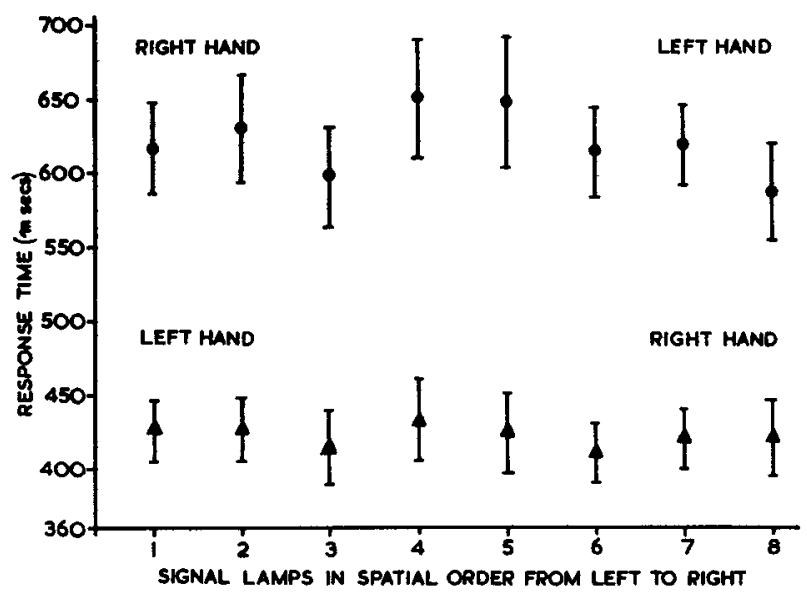

Fig. 1.

Lamps was also significant. Evidently lamps nearer the center of the display are more difficult to locate than are lamps at the extreme ends, and the effects of signal discriminability interact with the effects of S-R compatibility.

\section{Discussion}

Earlier studies have shown that the effects of S-R compatibility interact with the effects of variations in the size of the response ensemble (Griew, 1958), with the effects of response bias (Broadbent \& Gregory, 1965), with the effects of time uncertainty (Broadbent \& Gregory, 1965), and with the effects of response repetition (Bertelson, 1962). It is also known that signal discriminability interacts with variations in the response ensemble (Crossman, 1955; Rabbitt, 1963). In this context the present result fills in a picture of interactions between a number of different variables affecting choice-response time. Among other possible functional models it eliminates the possibility that in this particular task Ss make mediating responses in order to code signals on the display (Sternberg, 1964).

Various functional models may be postulated to explain these interactions. For example, operations necessary for the perceptual identification of a signal and the organization of an appropriate resporse may be described as more or less complex "codes" for the translation of signals into responses. If any part of a "code" is elaborated, the whole may become more vulnerable to the effects of "noise" in the CNS. An implied assumption is that the level of CNS "noise" varies randomly over time, so that any increase in the duration of a sequence of CNS events will also mean an increased probability that one or more of them will be affected by a local increase in noise level. The interaction effects we have discussed can only be explained in terms of this model if we make the further assumption that the result of interference by CNS noise is an increase in the time taken to perform a given operation or series of operations. Recent models for choice RT (e.g., Stone, 1960; Laming, 1963) make such a prediction, but themselves suggest an alternative line of explanation. Stone and Laming postulate that Ss may increase the probability of a correct signal identification by increasing the time over which they sample perceptual evidence. A given sampling time is supposed to be optimal for a given task, depending on the mutual discriminability of signals and on the number of signals to be distinguished. It is also possible that sampling time may vary with the relative payoffs for speed and accuracy in a given task. Thus, variations in $S-R$ compatibility may affect the confidence with which the $\mathrm{S}$ approaches the task as a whole-and so influence him to adopt more or less conservative criteria in deciding what is the optimal sampling period.

The present data share a defect of earlier results in that they were obtained from naive, unpracticed Ss. The relative contributions of all the variables discussed differ with the level of practice at which performance is sampled, and it is probable that the nature of the interactions between them will also vary with S's experience of the task. In the long view the existence of these interactions may be less crucial to the understanding of compatibility effects than is the order in which they disappear with extended practice.

\section{References}

Bertelson, P. S-R relationships and reaction times to new versus repeated signals in a serial task. J. exp. Psychol., 1963, 65, 478-484.

Broadbent, D. E., \& Gregory, Margaret. On the interaction of $S-R$ compatibility with other variables affecting reaction time. Brit. J. Psychol., 1965, 56, 61-67.

Egeth, H. E. Parallel vs, serial processes in multidimensional stimulus discrimination. Percept. \& Psychophys., 1966, 1, 245252.

Fitts, P., \& Switzer, G. Cognitive aspects of information processing: I. The familiarity of $\mathrm{S}-\mathrm{R}$ sets and subsets. J. exp. Psychol., 1962, 63, 321-329.

Griew, S. Information-gain in tasks involving different stimulusresponse relationships. Nature, 1958, 182, 1819.

Hick, W. E. On the rate of gain of information. Quart. J. exp. Psychol., 1952, 4, 11-26.

Laming, D. R. J. Human choice reaction-times. Unpublished Ph.D. dissertation, University of Cambridge, 1963.

Rabbitt, P. M. A. Information-load and discriminability. Nature, $1963,197,726$.

Stemberg, S. Two operations in character recognition: Some evidence from reaction-time measurements. Paper presented at the A.F.C.R.L. Symposium on models for the perception of speech and visual form. Boston, Mass., 11-14 No. 1964.

Stone, M. Models for choice reaction-time. Psychometrika, 1960 25, 251-260.

\section{Note}

1. A. Davidson, R. Olson, G. Baigent and F. Roberts designed and built SPARTA and carried out the testing. 